\title{
Old dilemma: asthma with irreversible airway obstruction or COPD
}

\author{
Fatemeh Fattahi $i^{1,2,3}$ • Judith M. Vonk ${ }^{3,4}$ - Nicole Bulkmans ${ }^{5}$ - Ruth Fleischeuer ${ }^{6}$. \\ Annette Gouw ${ }^{2,3}$ • Katrien Grünberg ${ }^{7}$ - Thais Mauad $^{8}$ • Helmut Popper ${ }^{9}$. \\ Aloisio Felipe-Silva ${ }^{8} \cdot$ Bart Vrugt $^{10}$ • Joanne L. Wright ${ }^{11}$ - Hui-Min Yang ${ }^{11}$. \\ Janwillem W.H. Kocks ${ }^{3,12}$ • Machteld N. Hylkema ${ }^{2,3}$. Dirkje S. Postma ${ }^{1,3}$. \\ Wim Timens $^{2,3}$ • Nick H. T. ten Hacken ${ }^{1,3}$
}

Received: 25 June 2015 / Revised: 28 July 2015 / Accepted: 4 August 2015 / Published online: 14 September 2015

(C) The Author(s) 2015. This article is published with open access at Springerlink.com

\begin{abstract}
Older asthmatic patients may develop fixed airway obstruction and clinical signs of chronic obstructive pulmonary disease (COPD). We investigated the added value of pathological evaluation of bronchial biopsies to help differentiate asthma from COPD, taking
\end{abstract}

Wim Timens and Nick H. T. ten Hacken shared last authorship.

Electronic supplementary material The online version of this article (doi:10.1007/s00428-015-1824-6) contains supplementary material, which is available to authorized users.

Wim Timens

w.timens@umcg.nl

Nick H. T. ten Hacken

n.h.t.ten.hacken@umcg.nl

Fatemeh Fattahi

f.fattahi@umcg.nl

Judith M. Vonk

j.m.vonk@umcg.nl

Nicole Bulkmans

n.bulkmans@kg.nl

Ruth Fleischeuer

r.fleischeuer@elisabeth.nl

Annette Gouw

a.s.h.gouw@umcg.nl

Katrien Grünberg

k.grunberg@vumc.nl

Thais Mauad

tmauad@usp.br

Helmut Popper

helmut.popper@medunigraz.at

Aloisio Felipe-Silva

aloisio_silva@ig.com.br into account smoking, age, and inhaled corticosteroid (ICS) use. Asthma and COPD patients (24 of each category) were matched for ICS use, age, FEV $\mathrm{F}_{1}$, and smoking habits. Five pulmonary and five general pathologists examined bronchial biopsies using an interactive
Bart Vrugt

bart.vrugt@usz.ch

Joanne L. Wright

jlwright@mail.ubc.ca

Hui-Min Yang

huiminyang@hotmail.com

Janwillem W.H. Kocks

j.w.h.kocks@umcg.nl

Machteld N. Hylkema

m.n.hylkema@umcg.nl

Dirkje S. Postma

d.s.postma@umcg.nl

Department of Pulmonology, University of Groningen, University Medical Center Groningen, PO Box 196, 9700

AD Groningen, The Netherlands

2 Department of Pathology and Medical Biology, University of Groningen, University Medical Center Groningen,

Groningen, The Netherlands

3 Research Institute for Asthma and COPD (GRIAC), University of Groningen, University Medical Center Groningen,

Groningen, The Netherlands 
website, without knowing patient information. They were asked to diagnose asthma or COPD on biopsy findings in both a pairwise and randomly mixed order of cases during four different phases, with intervals of 4-6 weeks, covering a maximal period of 36 weeks. Clinically concordant diagnoses of asthma or COPD varied between $63 \%-73 \%$, without important differences between pairwise vs randomly mixed examination or between general vs pulmonary pathologists. The highest percentage of concordant diagnoses was in young asthmatic patients without ICS use and in COPD patients with ICS use. In non ICS users with fixed airway obstruction, a COPD diagnosis was favored if abnormal presence of glands, squamous metaplasia, and submucosal infiltrate was present and an asthma diagnosis in case of abnormal presence of goblet cells. In ICS users with fixed airway obstruction, abnormal presence of submucosal infiltrates, basement membrane thickening, eosinophils, and glands was associated with asthma. Histological characteristics in bronchial biopsies are reproducibly recognized by pathologists, yet the differentiation by histopathology between asthma and COPD is difficult without information about ICS use.

Keywords Asthma $\cdot$ COPD $\cdot$ Asthma COPD Overlap Syndrome $\cdot$ Pathology

\section{Introduction}

Asthma and chronic obstructive pulmonary disease (COPD) are heterogeneous chronic lung diseases, characterized by the presence of airway obstruction and

\footnotetext{
$\overline{4}$ Department of Epidemiology, University of Groningen, University Medical Center Groningen, Groningen, The Netherlands

5 Department of Pathology, Spaarne Gasthuis Haarlem-Zuid, Haarlem, The Netherlands

6 Department of Pathology, Elisabeth-TweeSteden Ziekenhuis, Tilburg, The Netherlands

7 Department of Pathology, VU Medical Center, Amsterdam, The Netherlands

8 Department of Pathology, Sao Paulo University, Sao Paulo, Brazil

9 Institute of Pathology, Research Unit Molecular Lung and Pleura Pathology, Medical University Graz, Graz, Austria

10 Department of Surgical Pathology, University of Zurich, Zurich, Switzerland

11 Department Pathology, University of British Columbia, Vancouver, B.C., Canada

12 Department of General Practice, University of Groningen, University Medical Center Groningen, Groningen, The Netherlands
}

airway inflammation $[1,2]$. In asthma, airway obstruction is typically completely or nearly completely reversible [2], while irreversible airway obstruction is typical for COPD [1]. Although both diseases have overlapping clinical features, acknowledged in guidelines [3], they are generally regarded as different disorders; each requiring their own diagnostic and management strategies. For example, for the pharmacological management of asthma, inhaled corticosteroids (ICSs) are the most efficacious drugs currently available [2]. In COPD, inhaled bronchodilators are fundamental for treatment, whereas addition of ICS is only recommended for COPD patients suffering from severe disease $\left(\mathrm{FEV}_{1}<50 \%\right.$ predicted $)$ and/or a history of recurrent exacerbations [1]. In order to determine appropriate treatment, prognosis and follow-up, international guidelines have emphasized the importance of differentiating asthma from COPD.

In general, careful history taking, physical examination, and lung function testing often lead to a clear diagnosis [4]. However, it is frequently difficult if not impossible to achieve an accurate diagnosis of either asthma or COPD in older patients [5-8]. Although asthma generally affects children and young adults, it is not uncommon that asthma starts later in life [9]. In approximately 4 to $8 \%$ of asthmatic cases, the first asthma symptoms are present in late adulthood (late-onset asthma) or even after 65 years of age. With increasing age, a proportion of patients with asthma may develop persistent irreversible airflow limitation, particularly in the presence of risk factors such as smoking [5], blood eosinophilia, chronic mucus hypersecretion, and a low level of $\mathrm{FEV}_{1}$ [10]. This type of asthma is clinically indistinguishable from COPD, which the guidelines called ACOS or asthma-COPD overlap syndrome [3], and medical history, physical examination, and lung function tests may become insufficient to distinguish asthma from COPD to allow the most adequate therapy. The lack of a diagnostic standard to identify asthma at older age, together with poor perception of symptoms such as dyspnea, may further hamper the recognition of asthma in the elderly [11].

In case of doubt, clinicians may attempt to achieve a best possible diagnosis of asthma or COPD by taking bronchial biopsies for histopathological examination, although this is not a common practice. It has been suggested that pathological examination of bronchial tissue, taking features such as denudation of the epithelium in asthma and epithelial hyperplasia in COPD into account, might contribute to resolving the diagnostic difficulty. Despite clear morphological differences between asthma and COPD, some morphological characteristics can be found in both diseases (in particular 
in chronic or severe cases), which impairs their diagnostic value in an individual case [12]. Bourdin et al. demonstrated that the diagnostic value of histological examination of endobronchial biopsies from subjects with asthma or COPD is limited, sensitivity and specificity ranging between 36-48 and 56-79\% respectively [13]. However, the latter study included mostly young, never-smoking, non-steroid-using asthma patients with normal lung function and high bronchodilator reversibility. Furthermore, asthma and COPD patients were not matched for age, airway obstruction, ICS use, and smoking habits. Since these factors modulate histological features of airway inflammation and remodeling, they therefore may have confounded the results.

In the current study, we aimed to identify the most important histopathological features to differentiate between asthma and COPD in bronchial biopsies. We hypothesized that the accuracy of the pathological diagnosis would improve when taking into account modulating factors such as smoking, age, and ICS use.

\section{Materials and methods}

\section{Patients and matching of biopsies}

Biopsies from 24 asthma and 24 matched (see below) COPD patients were included. Subjects were only included when there was no uncertainty in the diagnosis and when subjects met all criteria for either asthma or COPD according to international guidelines $[1,2]$. Subjects were selected from several asthma and COPD cohort studies performed in our institute [14-17]. We created three groups $(\mathrm{A}-\mathrm{C})$ of asthma patients $(n=8$ each group), carefully matched with three groups of COPD patients ( $n=8$ each group).

A. The first asthma and COPD groups included subjects who did not use ICS, were $>45$ years old, had a postbronchodilator (BD) $\mathrm{FEV}_{1} / \mathrm{FVC}<70 \%$, and had smoked $>10$ pack-years.

B. The second asthma and COPD groups included subjects with the same criteria, but subjects had used ICS during the last 30 months.

C. The third group included asthma patients without ICS use, and with post-BD $\mathrm{FEV}_{1}>90 \%$ predicted, age $<45$ years, 0 pack-years smoking, and atopy (Phadiatop > 1.0). This was contrasted with COPD patients without ICS use, with post-BD $\mathrm{FEV}_{1}<50 \%$ predicted, age $>45$ years, current smoking with $>10$ packyears, and without atopy.

Groups A and B included patients with a clinically difficult differential diagnosis between asthma and COPD, whereas control group $\mathrm{C}$ included so-called classical cases easy to differentiate.

Table 1 shows the group characteristics $(\mathrm{A}-\mathrm{C})$ and Table S1 individual characteristics. Details of the selection process are depicted in the Fig. S1.

Medical records, lung function data, and paraffinembedded endobronchial biopsies (EBB) were available from all selected patients.

\section{EBB staining, virtual microscopy, and interactive website}

EBB slides were stained with hematoxylin and eosin (H\&E). An experienced pulmonary pathologist (WT) checked the quality of the slides for each patient and selected the best specimen based on size, intactness, and presence of mucosal

Table 1 Characteristics of patients with asthma and COPD

\begin{tabular}{|c|c|c|c|c|c|c|}
\hline \multirow[b]{2}{*}{ Characteristics } & \multicolumn{2}{|l|}{$\begin{array}{l}\text { Group A } \\
\text { (ICS-) }\end{array}$} & \multicolumn{2}{|l|}{$\begin{array}{l}\text { Group B } \\
(\mathrm{ICS}+)\end{array}$} & \multicolumn{2}{|c|}{$\begin{array}{l}\text { Group C } \\
\text { (Classical, ICS-) }\end{array}$} \\
\hline & Asthma & COPD & Asthma & COPD & Asthma & COPD \\
\hline Age, years & $53(50-64)$ & $56(47-63)$ & $61(54-68)$ & $61.5(56-72)$ & $29.5(25-44)$ & $63(53-64)$ \\
\hline Sex, M/F & $6 \mathrm{M}, 2 \mathrm{~F}$ & $5 \mathrm{M}, 3 \mathrm{~F}$ & $5 \mathrm{M}, 3 \mathrm{~F}$ & $8 \mathrm{M}, \mathrm{OF}$ & $4 \mathrm{M}, 4 \mathrm{~F}$ & $6 \mathrm{M}, 2 \mathrm{~F}$ \\
\hline $\mathrm{FEV}_{1} / \mathrm{FVC}, \%$ & $64(48-69)$ & $62(48-70)$ & $53(40-66)$ & $53(36-69)$ & $81(75-98)$ & $41(30-47)$ \\
\hline $\mathrm{FEV}_{1}, \%$ pred & $83(60-108)$ & $82(70-106)$ & $83(43-99)$ & $71(46-90)$ & $105(95-122)$ & $45(41-50)$ \\
\hline Pack-years & $31(10-44)$ & $29(15-43)$ & $20(12-64)$ & $38(19-51)$ & $0.0(0.0-0.0)$ & $32(21-56)$ \\
\hline Current smoking, $n$ ex smoking, $n$ & 8 current, 0 ex & 8 current, 0 ex & 6 current, 2 ex & 6 current, 2 ex & 0 current, 0 ex & 8 current, 0 ex \\
\hline
\end{tabular}

Data presented as median (minimum-maximum) except for sex ( $M$ male, $F$ female) and smoking (current, ex smoking). Group A: asthma and COPD patients without ICS use, age $>45$ years, post bronchodilator (BD) $\mathrm{FEV}_{1} / \mathrm{FVC}<70 \%$, and $>10$ pack-years smoking. Group $\mathrm{B}$ : asthma and COPD patients with the same criteria, but subjects had to use ICS during last 30 months. Group C: "classical" asthma patients without ICS use, and with post BD $\mathrm{FEV}_{1}>90 \%$ predicted, age $<45$ years, 0 pack-years smoking, and atopy. Classical asthma was contrasted with classical COPD: no ICS use, post BD $\mathrm{FEV}_{1}<50 \%$ predicted, age $>45$ years, current smoking with $>10$ pack-years, and no atopy 
Fig. 1 Example slide on interactive website. Screenshot of the interactive website, showing a slide with a representative bronchial biopsy at $\times 20$ magnification. The small image in the lower part of the picture is the overview window, showing the current position and size of the large window. The website allowed to view the slides at different magnifications and to navigate into different areas of the bronchial biopsy like a normal microscope

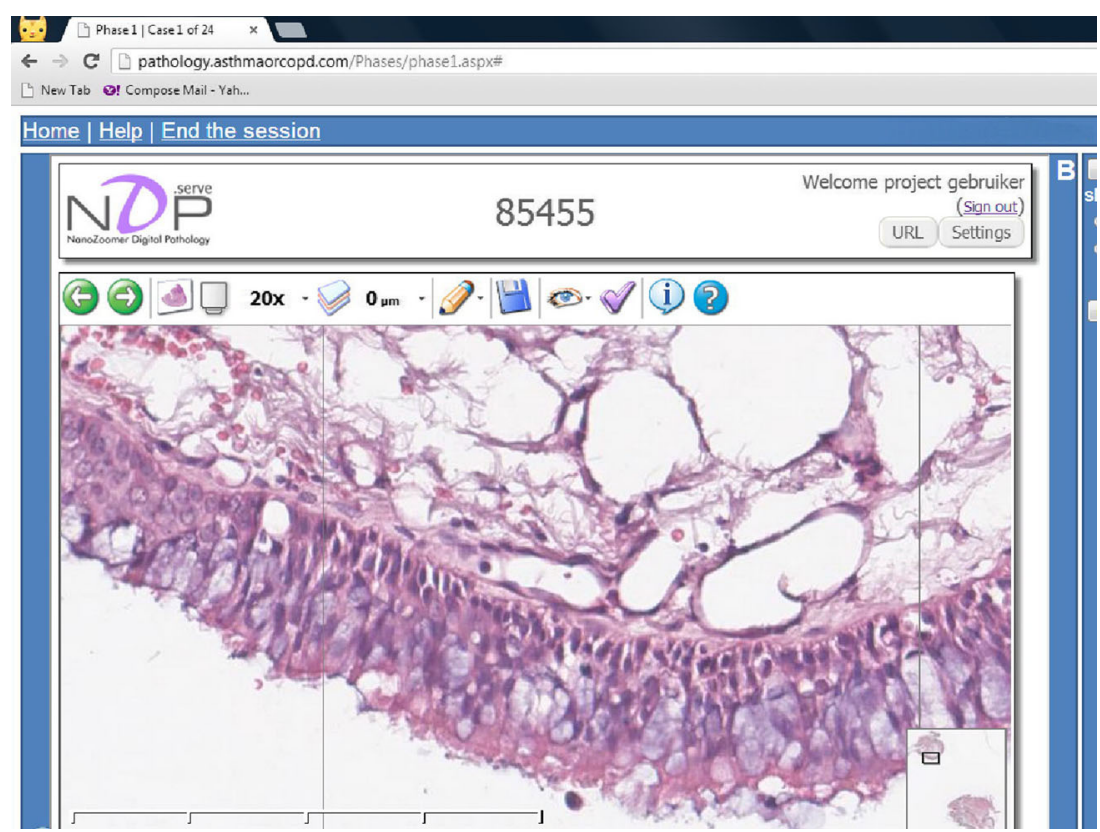

and submucosal layers. This quality check was performed without knowing the diagnosis or paying attention to the possible diagnosis. Entire biopsies were scanned using Aperio ScanScope Digital Slide Scanner at $\times 40$ magnification. Afterwards, the images were uploaded to a specially designed interactive website, allowing to view the slides at different magnifications and to navigate into different areas of the bronchial biopsy like with a normal microscope (Fig. 1). Quality of our web-based virtual microscopy was checked by an experienced independent pulmonary pathologist who compared all 48 slide images with series of biopsies from the original glass slides by microscope.

Table 2 Design of the study

\begin{tabular}{|c|c|c|c|}
\hline & Time (weeks) & Examination of slides & Description \\
\hline Phase 1 & 2 & Pairwise $(2 \times 24$ slides $)$ & $\begin{array}{l}\text { Matched asthma and COPD slides were offered pairwise. The pathologists were informed } \\
\text { about this and could only opt for asthma or COPD, thus chose for two options per pair. } \\
\text { Once chosen, the pathologists were not able to change their choice or go back to slides } \\
\text { shown earlier. }\end{array}$ \\
\hline Interval & $4-6$ & & \\
\hline Phase 2 & 4 & Randomly mixed (48 slides) & $\begin{array}{l}\text { The } 48 \text { slides were randomly mixed. The pathologists were informed about this and had to } \\
\text { opt for asthma or COPD per slide. Once chosen, the pathologists were not able to change } \\
\text { their choice or go back to slides shown earlier. }\end{array}$ \\
\hline Interval & $4-6$ & & \\
\hline Phase $3 \mathrm{a}$ & 2 & $\begin{array}{l}\text { Randomly mixed + criterion } \\
\text { list (48 slides) }\end{array}$ & $\begin{array}{l}\text { Conform phase } 2 \text {. Additionally, the pathologists were asked per slide to score for the } \\
\text { presence or absence of a criterion that drove their diagnosis (Box 1). After scoring the } \\
\text { criterion list they had to choose for asthma or COPD and to give their level of certainty } \\
\text { (VAS 0-10). }\end{array}$ \\
\hline Interval & $4-6$ & & \\
\hline Phase $3 b$ & 2 & $\begin{array}{l}\text { Randomly mixed + criterion } \\
\text { list ( } 24 \text { slides })\end{array}$ & Conform phase $3 \mathrm{a}$. This phase aimed to test repeatability. \\
\hline Interval & $4-6$ & & \\
\hline \multirow[t]{2}{*}{ Phase 4} & 2 & Pairwise $(2 \times 24$ slides $)$ & Conform phase 1 . This phase aimed to test repeatability (and/or potential learning effects). \\
\hline & $\begin{array}{l}\text { Totally } 28- \\
36 \text { weeks }\end{array}$ & & \\
\hline
\end{tabular}




\section{Study protocol}

Ten pathologists from different countries (Netherlands, Brazil, Canada, Austria, Switzerland), five of them specialized in pulmonary pathology, participated in the study and used our interactive website following a strict protocol (Table 2). The pathologists were informed about the design of the study but had no clinical information (age, sex, ICS use, smoking, lung function). In phases 1 and 4, the pathologists were offered 48 slides, i.e., 24 pairs of matched asthma and COPD patients, and were asked to indicate per pair which one was asthma and which one COPD. In phases 2 and 3, all 48 slides were offered in a randomly mixed order and the pathologists were asked to choose for either asthma or COPD. In phase 3, the pathologists were additionally asked to indicate the presence or absence of a pathological criterion using the criteria list of Bourdin's study [13], with small modifications (Box 1). In addition, they were asked how sure they felt about their diagnosis using a $0-10$ visual analog scale (VAS) score $(0$ not sure, 10 very sure) and to rank what they considered the three most relevant features (Fig. 2).

Box 1

\section{Questionnaire for pathologists}

Indicate per criterion if it is abnormally (disease-related) present (yes/no)

\section{Epithelium}

- Denudation (loss of epithelium, including loss of super-basal epithelium)

- Squamous metaplasia

- Hyperplasia (=thickened epithelium)

- Goblet cells

\section{Basement membrane}
- Thickening

\section{Submucosa}

- Inflammatory infiltrate (lymphocytes, macrophages or neutrophils)

- Eosinophils

- Smooth muscle

- Glands

- Sub-mucosal fibrosis

\section{Diagnosis:}

- Asthma or COPD?

- How sure are you about the diagnosis (score 1-10):

- Which criterion was most important for your diagnosis?

First:

Second:

Third:

\section{Statistical analyses}

Data from the interactive website were automatically and anonymously saved to an Excel file (MS Excel 2010) and transferred to SPSS software (version 19.0; SPSS Inc., Chicago, IL). The individual data was computed to a concordant or discordant diagnosis of asthma or COPD (i.e., concordant between pathological and clinical diagnosis), and results were expressed as the mean percentage of the concordant diagnosis. In phase 3 , the reported presence of each pathological criterion was compared between 
slides of asthma and COPD using the Mann-Whitney test. Sensitivity, specificity, and accuracy of each pathological criterion for pathologists making a concordant diagnosis of asthma were calculated. A high sensitivity for asthma indicated automatically a high specificity for COPD and vice versa. Pathological criteria with a $p$ value $<0.2$ in the univariate analysis were entered in a logistic regression analysis on the presence of asthma. Pathological criteria which contributed independently to a concordant diagnosis of asthma or COPD were combined to find higher accuracy rates for the concordant diagnosis. Selection of combined criteria was based on the highest Wald value in the regression analyses.

Analyses were performed in the total group of patients, within patient groups $(\mathrm{A}-\mathrm{C}$ ), and between pathologists (general, specialized). Intra- (between phases $3 a$ and $3 b$, see Box 1) and inter-observer (within phase $3 a$ ) agreements were assessed with Cohen's kappa test and Fleiss' kappa, respectively. The significance level was set at 0.05 .

\section{Results}

\section{Concordant diagnoses (pathology concordant with clinical diagnosis)}

Table 3 shows the percentage of concordant diagnoses per phase, for each disease group (A-C) per pathologist group. The percentages of concordant diagnoses, per pathologist, per phase, are shown in Fig. S2. The percentage of concordant diagnoses of asthma or COPD, per pathologist group, in phase $3 \mathrm{a}$ is shown in Table S2. Overall, the highest number of concordant diagnoses was observed in phase 4 , particularly by pulmonary pathologists. The highest score for asthma was observed in phase $3 \mathrm{a}$, in the classical asthma/COPD group (C), by pulmonary pathologists, $91.4 \%$. The highest score for COPD was also observed in phase $3 \mathrm{a}$, in the non-classical asthma/COPD group of ICS users (B), with no difference between pulmonary and general pathologists. Feeling sure about the diagnosis of asthma (in asthma and COPD cases together) was rated on a VAS scale from 1 to 10. In groups A-C, the mean (SD) VAS score for asthma was 5.6 (2.5), 5.2 (2.0), and 6.1 (2.4), respectively. For COPD, this was 5.9 (2.4), 5.8 (2.1), and $5.0(2.6)$.

\section{Pathological criteria in phase 3}

Table 4 shows the reported presence of pathological criteria in the airway wall biopsies in asthma or COPD per disease group $(4 \mathrm{~A}-\mathrm{C})$. Criteria that differed significantly between asthma and COPD were not comparable between the three groups. Eosinophilia was significantly more frequently reported in asthma in groups $\mathrm{B}+\mathrm{C}$ but not in A (subjects with asthma and COPD in B used ICS, in contrast to subjects in A $+\mathrm{C}$ ). Submucosal inflammation was significantly more frequently reported in asthma in groups $\mathrm{B}+\mathrm{C}$ than in group $\mathrm{A}$, yet more frequently in COPD in group A. Table S3 and Box 2 show which pathological criteria significantly contribute to a concordant diagnosis of asthma or COPD in a multiple regression
Fig. 2 Example slide on interactive website including the list with pathological criteria. Screenshot of the interactive website, showing a slide (phase $3 a)$ with a representative bronchial biopsy at $\times 10$ magnification. The small image in the lower part of the picture is the overview window, showing the current position and size of the large window. At the right side of the slide the pathologists may record the observed abnormal presence of 12 pathological criteria (yes/no), the diagnosis of asthma or COPD, how sure they feel about the diagnosis, and rank the 3 most relevant features

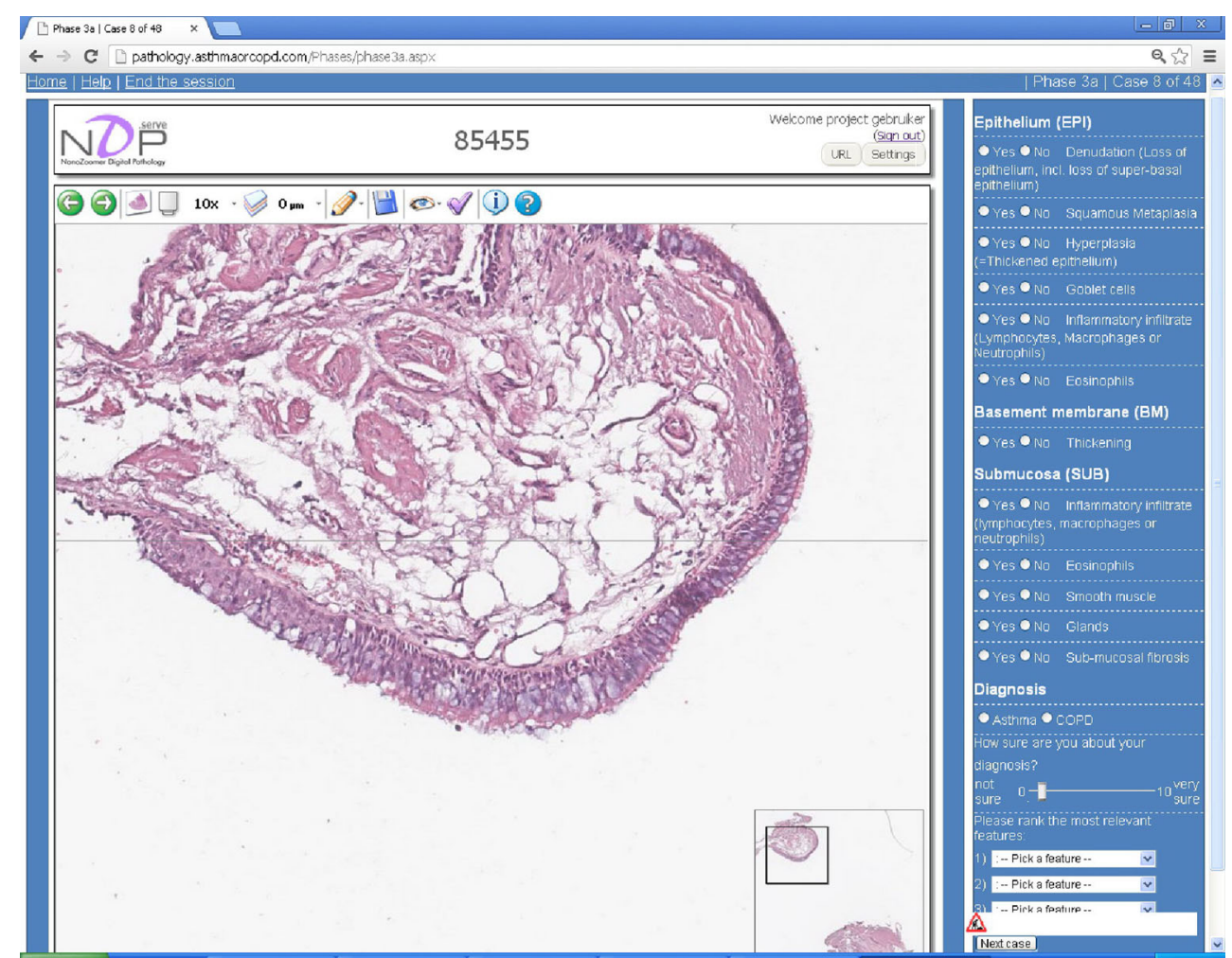


model. Significant criteria present in all groups were goblet cells, inflammatory infiltrate, and glands. Significant criteria present in two groups were eosinophils (group B $+\mathrm{C}$ ), and in one group squamous metaplasia (group A), BM thickening (group B), hyperplasia (group C), smooth muscle (group C), and submucosal fibrosis (group C). Significant $(p<0.05$ ) Wald values of these models are also shown in Table 4.

\section{Sensitivity, specificity, and accuracy of criteria for a concordant diagnosis}

Table 4 shows the sensitivity, specificity, and accuracy for asthma/COPD diagnosis for groups A-C. Pathological criteria with high accuracy differed importantly between groups A-C. In group A (no ICS use), glands, goblet cells, squamous metaplasia, and submucosal infiltrate showed the highest accuracy. In group B (with ICS use), the highest accuracy was provided by submucosal inflammation, basement membrane thickening, eosinophilia, and glands. In group C (classical asthma and COPD), the highest accuracy was shown by submucosal fibrosis, epithelial hyperplasia, eosinophilia, and glands. Combinations of relevant pathological criteria did improve sensitivity or specificity, but in general not accuracy (Table S4).

\section{Agreement between and repeatability within pathologists}

Agreement between pathologists for a concordant diagnosis of asthma or COPD in groups A-C was $0.40,0.45$, and 0.48 , respectively. This was $0.33,0.45$, and 0.72 for asthma and $0.60,0.57$, and 0.27 for COPD, respectively. Repeatability within pathologists for a concordant diagnosis in groups A$\mathrm{C}$ was $0.75,0.78$, and 0.80 respectively. This was $0.75,0.70$, and 0.80 for asthma and $0.70,0.85$, and 0.80 for COPD, respectively. Agreement between and repeatability within pathologists for the reported presence of pathological criteria in groups $\mathrm{A}-\mathrm{C}$ is presented in Table 4. Overall agreement in groups $\mathrm{A}-\mathrm{C}$ varied between 0.61 and 0.92 . Overall repeatability in groups $\mathrm{A}-\mathrm{C}$ varied between 0.68 and 0.95 .

\section{Discussion}

Asthma and COPD are obstructive airway diseases with clear differences in etiology and pathophysiology, yet at older age, they frequently are difficult to discriminate, the currently socalled asthma-COPD overlap syndrome (ACOS) [3]. In the current study, there was no discussion about the original underlying disease, as we had historical data from wellcharacterized cohort studies. Therefore, we had the opportunity to carefully match bronchial biopsies from 24 asthma and 24 COPD patients taking age, $\mathrm{FEV}_{1}$, ICS use, and smoking habits into account. Ten pathologists, not informed about the individual clinical background of the patients, but knowing the study design, were asked to diagnose asthma or COPD. The important outcome of this study is that histological examination of bronchial biopsies alone does not allow differentiating between asthma and COPD. However, as recognition of the specific histological criteria was good, diagnostic value can be expected to improve when selected pathological criteria are applied and adequate clinical information is provided, in which in particular, knowledge about the use of ICS is essential.

In this study, we aimed for high-quality matching of asthma and COPD patients, considering age, smoking, ICS use, and airway obstruction as important modulators of airway inflammation and remodeling. This matching is important because it fits with the original question of the study: whether it is possible for pathologists to discriminate between older asthma patients with fixed airway obstruction and COPD patients, using bronchial biopsies. We designed our study into four phases, gradually increasing the level of difficulty and anticipated that a head-to-head comparison of two paired slides, one from an asthma patient and one from a COPD patient, would help to discriminate between the two diseases. Interestingly, this was not the case. Success rates of scoring of the paired slides were not higher than those of randomly mixed slides. Furthermore, we anticipated that systematic scoring of textbook pathological criteria for asthma or COPD would help to set the correct (=concordant) diagnosis; however, this was not the case.

The overall percentages of a diagnosis of asthma or COPD concordant with the clinical diagnosis were low, taking into account a $50 \%$ concordance by chance. We expected the highest percentage of concordant diagnoses in young asthmatic patients without ICS use and with a normal lung function, assuming that the recognition of the underlying disease is easier when inflammation is not treated with ICS or changed by age- and smoking-related remodeling processes. Indeed, the highest percentage of a concordant diagnosis for asthma in the randomly mixed slides was observed in the group of classical cases (group C, without ICS) and the lowest in the group of asthma subjects who used ICS clinically which are difficult to diagnose (group B). Interestingly, this contrasted with the findings for COPD, where the highest percentage of concordant diagnoses occurred in the group of ICS users. It is not clear at first sight why ICS use reduced the percentage of concordant diagnosis for asthma, yet improved this for COPD. This may well be because corticosteroids reduce eosinophil survival but prolong neutrophil survival; hence, ICS use may have reduced eosinophilia in asthma and increased neutrophilia in COPD [18], eosinophilia being considered a hallmark for asthma and neutrophilia for COPD. This contrasting effect of ICS use on giving a concordant diagnosis 
Table 3 Percentage of concordant diagnoses in different phases of the study

\begin{tabular}{|c|c|c|c|c|c|c|c|}
\hline & & \multirow{2}{*}{$\begin{array}{l}\text { Phase } 1 \\
\text { Pairwise }\end{array}$} & \multicolumn{2}{|l|}{ Phase 2} & \multicolumn{2}{|c|}{ Phase $3 a$} & \multirow{2}{*}{$\begin{array}{l}\text { Phase } 4 \\
\text { Pairwise }\end{array}$} \\
\hline & & & Asthma & COPD & Asthma & COPD & \\
\hline \multirow[t]{4}{*}{ All pathologists } & All & 68.7 & 65.6 & 62.5 & 63.5 & 62.5 & 72.6 \\
\hline & A (ICS-) & 65.0 & 67.5 & 62.5 & 56.3 & 65.0 & 63.8 \\
\hline & $\mathrm{B}(\mathrm{ICS}+)$ & 73.8 & 52.5 & 66.2 & 52.5 & 72.5 & 76.3 \\
\hline & $\mathrm{C}$ (classical) & 67.1 & 78.6 & 58.7 & 84.3 & 50.0 & 78.6 \\
\hline \multirow[t]{4}{*}{ Lung pathologists } & All & 71.3 & 66.1 & 60.0 & 70.4 & 61.7 & 76.5 \\
\hline & A (ICS-) & 62.5 & 67.5 & 57.5 & 67.5 & 57.5 & 62.5 \\
\hline & B (ICS+) & 80.0 & 52.5 & 65.0 & 55.0 & 72.5 & 85.0 \\
\hline & C (classical) & 71.4 & 80.0 & 57.5 & 91.4 & 55.0 & 82.9 \\
\hline \multirow[t]{4}{*}{ General pathologists } & All & 66.1 & 65.2 & 65.0 & 56.5 & 63.3 & 68.7 \\
\hline & A (ICS-) & 67.5 & 67.5 & 67.5 & 45.0 & 72.5 & 65.0 \\
\hline & $\mathrm{B}(\mathrm{ICS}+)$ & 67.5 & 52.5 & 67.5 & 50.0 & 72.5 & 67.5 \\
\hline & $\mathrm{C}$ (classical) & 62.9 & 77.1 & 60.0 & 77.1 & 45.0 & 74.3 \\
\hline
\end{tabular}

Values are percentage of concordant diagnoses. Italic values: $p<0.05$ between $\mathrm{A}, \mathrm{B}$, and $\mathrm{C}$ within $3 \mathrm{a}$ phase. Group A: asthma and COPD patients without ICS use, age $>45$ years, post bronchodilator (BD) $\mathrm{FEV}_{1} / \mathrm{FVC}$ $<70 \%$, and $>10$ pack-years smoking. Group B: asthma and COPD patients with the same criteria, but subjects had to use ICS during last 30 months. Group C: "classical" asthma patients without ICS use, and with post BD $\mathrm{FEV}_{1}>90 \%$ predicted, age $<45$ years, 0 pack-years smoking, and atopy. Classical asthma was contrasted with classical COPD: no ICS use, post BD FEV ${ }_{1}<50 \%$ predicted, age $>45$ years, current smoking with $>10$ packyears, and no atopy of asthma or COPD is an important finding of our study, which - it goes without saying - emphasizes the need for effective transfer of adequate clinical data from clinicians to pathologists.

The reported presence of pathological criteria was significantly different between slides from asthma and COPD patients, in striking contrast with the low percentage of concordant diagnoses of asthma and COPD based on biopsies alone. Additionally, the agreement between the 10 pathologists (interobserver variability) and repeatability within pathologists (intra-observer variability) was good to excellent for many pathological criteria, independent of a concordant diagnosis. Thus, all expertise to allow a concordant diagnosis of asthma or COPD was present, yet the pathologists appeared to need additional information to be able to apply this expertise successfully, considering the relatively low percentage of concordant diagnoses. A priory, one might expect that the diagnosis of pathologists based upon histology only might be more accurate because it allows integration of all relevant information from all parts of a biopsy. Apparently, this is a difficult task, which is in particular complicated when clinical characteristics like age, smoking, ICS use, and airway obstruction, that affect accuracy and significance of potentially useful pathological criteria, are not known, as shown in the present study.

Next, we tried to identify pathologists with a higher percentage of concordant diagnoses in order to learn and copy their strategy. Beforehand, we expected that pulmonary pathologists would do better than general pathologists. Indeed, at a group level, the mean scores of the pulmonary pathologist group tended to be higher in every phase of the study, and the pathologists with the highest percentages of concordant diagnoses were almost always the pulmonary pathologists. For example, one of the pulmonary pathologists (pathologist B in Table S2) had high accuracy rates, but importantly, in the subgroup of patients who did use ICS (group B), this pathologist had poor results, again demonstrating the importance of knowledge of the clinical background of patients. The pathological criteria that were most frequently ranked as important for the diagnosis by the best scoring pathologists were squamous metaplasia in group A (without ICS use), basement membrane thickening in group B (with ICS use), and submucosal fibrosis in group C (with "classical”" asthma and COPD). This is not unexpected as these pathological criteria demonstrated also high accuracy rates at a group level. Finally, we tried to improve accuracy rates by combining pathological criteria that independently associated with the clinical diagnosis of asthma or COPD. Whereas it was not difficult to find combinations with a very high sensitivity or specificity rates, the accuracy rates of these combinations were generally not better than the individual pathological criteria.

We hypothesize that pathologists may improve their ability to differentiate asthma from COPD if they use selected pathological criteria, i.e., those with a high accuracy rate for concordance, provided that the relevant clinical information is known. In non ICS users with fixed airway obstruction the abnormal presence of goblet cells directs towards an asthma diagnosis, whereas glands, squamous metaplasia, and submucosal infiltrate direct towards COPD (Box 2). In ICS users 
with fixed airway obstruction, the abnormal presence of submucosal infiltrate, basement membrane thickening, eosinophils, and glands direct towards asthma diagnosis. In classical cases, the diagnosis is already known on the basis of clinical characteristics. Nevertheless, the clinical diagnosis can be confirmed by the observation of submucosal infiltrate and eosinophils indicating asthma, whereas submucosal fibrosis, hyperplasia, and glands indicate COPD.

Table 4 Characteristics of the pathological criteria for the diagnosis of asthma or COPD in group A (non-ICS users), group B (ICS users), and group C (classical group)

\begin{tabular}{|c|c|c|c|c|c|c|c|c|c|}
\hline \multirow[t]{2}{*}{ Criteria } & \multicolumn{2}{|c|}{$\begin{array}{l}\text { Reported presence } \\
(\%) \text { in }\end{array}$} & \multirow[t]{2}{*}{$\begin{array}{l}\text { OR (asthma) } \\
(95 \% \mathrm{CI})\end{array}$} & \multirow[t]{2}{*}{$\begin{array}{l}\text { Wald } \\
\text { value }\end{array}$} & \multirow[t]{2}{*}{$\begin{array}{l}\text { Sensitivity } \\
(\%)\end{array}$} & \multirow[t]{2}{*}{$\begin{array}{l}\text { Specificity } \\
(\%)\end{array}$} & \multirow[t]{2}{*}{$\begin{array}{l}\text { Accuracy } \\
(\%)\end{array}$} & \multirow{2}{*}{$\begin{array}{l}\text { Agreement } \\
\text { between } \\
\text { pathologists }\end{array}$} & \multirow[t]{2}{*}{$\begin{array}{l}\text { Repeatability } \\
\text { within pathologis }\end{array}$} \\
\hline & Asthma & COPD & & & & & & & \\
\hline \multicolumn{10}{|l|}{ Group A (non-ICS users) } \\
\hline Denudation & 85.0 & 83.8 & $1.1(0.47-2.58)$ & & 85.0 & 16.3 & 50.6 & $0.76(0.77 ; 0.76)$ & $0.90(0.95 ; 0.85)$ \\
\hline Squamous Metaplasia & 22.5 & 53.8 & $0.25(0.13-0.49)$ & 7.74 & 77.5 & 53.8 & 65.6 & $0.72(0.77 ; 0.68)$ & $0.80(0.90 ; 0.70)$ \\
\hline Hyperplasia & 52.5 & 55.0 & $0.90(0.49-1.69)$ & & 47.5 & 55.0 & 51.2 & $0.63(0.73 ; 0.54)$ & $0.73(0.75 ; 0.70)$ \\
\hline Goblet cells & 60.0 & 32.5 & $3.11(1.63-5.95)$ & 5.54 & 60.0 & 67.5 & 63.7 & $0.85(0.87 ; 0.84)$ & $0.85(0.90 ; 0.80)$ \\
\hline BM thickening & 73.8 & 67.5 & $1.35(0.68-2.68)$ & & 73.8 & 32.5 & 53.1 & $0.67(0.69 ; 0.64)$ & $0.85(0.85 ; 0.85)$ \\
\hline Inflammatory infiltrate & 53.8 & 72.5 & $0.44(0.23-0.85)$ & 11.84 & 46.3 & 72.5 & 59.4 & $0.61(0.53 ; 0.69)$ & $0.78(0.60 ; 0.95)$ \\
\hline Eosinophils & 22.5 & 12.5 & $2.03(0.87-4.73)$ & & 22.5 & 87.5 & 55.0 & $0.72(0.64 ; 0.79)$ & $0.85(0.75 ; 0.95)$ \\
\hline Smooth muscle & 60.0 & 81.3 & $0.35(0.17-0.71)$ & & 40.0 & 81.3 & 60.6 & $0.76(0.78 ; 0.74)$ & $0.85(0.80 ; 0.90)$ \\
\hline Glands & 23.8 & 72.5 & $0.12(0.06-0.24)$ & 21.62 & 76.3 & 72.5 & 74.4 & $0.85(0.93 ; 0.76)$ & $0.90(1: 00 ; 0.80)$ \\
\hline Sub mucosal fibrosis & 67.5 & 58.8 & $1.46(0.76-2.78)$ & & 32.5 & 58.8 & 45.6 & $0.63(0.66 ; 0.60)$ & $0.75(0.85 ; 0.65)$ \\
\hline \multicolumn{10}{|l|}{ Group B (ICS users) } \\
\hline Denudation & 71.3 & 58.8 & $1.74(0.90-3.36)$ & & 71.3 & 41.3 & 56.2 & $0.62(0.61 ; 0.63)$ & $0.73(0.65 ; 0.80)$ \\
\hline Squamous metaplasia & 33.8 & 35.0 & $0.95(0.49-1.82)$ & & 66.3 & 35.0 & 50.6 & $0.80(0.80 ; 0.81)$ & $0.85(0.70 ; 1.00)$ \\
\hline Hyperplasia & 51.3 & 53.8 & $0.90(0.49-1.68)$ & & 48.8 & 53.8 & 51.2 & $0.67(0.71 ; 0.63)$ & $0.90(0.85 ; 0.95)$ \\
\hline Goblet cells & 72.5 & 60.0 & $1.76(0.90-3.41)$ & & 72.5 & 40.0 & 56.2 & $0.72(0.68 ; 0.76)$ & $0.60(0.60 ; 0.60)$ \\
\hline BM thickening & 57.5 & 25.0 & $4.06(2.07-7.95)$ & 18.24 & 57.5 & 75.0 & 66.2 & $0.71(0.78 ; 0.64)$ & $0.75(0.80 ; 0.70)$ \\
\hline Inflammatory infiltrate & 63.8 & 23.8 & $5.65(2.84-11.23)$ & 19.86 & 63.8 & 76.3 & 70.0 & $0.71(0.73 ; 0.70)$ & $0.83(0.80 ; 0.85)$ \\
\hline Eosinophils & 30.0 & 3.8 & $11.0(3.16-38.34)$ & 4.27 & 30.0 & 96.3 & 63.1 & $0.85(0.76 ; 0.93)$ & $0.83(0.70 ; 0.95)$ \\
\hline Smooth muscle & 66.3 & 61.3 & $1.24(0.65-2.37)$ & & 66.3 & 38.8 & 52.5 & $0.77(0.86 ; 0.68)$ & $0.80(0.80 ; 0.80)$ \\
\hline Glands & 48.8 & 33.8 & $1.87(0.99-3.53)$ & 8.88 & 48.8 & 66.3 & 57.5 & $0.81(0.79 ; 0.83)$ & $0.90(0.90 ; 0.90)$ \\
\hline Sub mucosal fibrosis & 66.3 & 67.5 & $0.94(0.49-1.83)$ & & 33.8 & 67.5 & 50.6 & $0.65(0.64 ; 0.66)$ & $0.78(0.85 ; 0.70)$ \\
\hline \multicolumn{10}{|l|}{ Group C (classical group) } \\
\hline Denudation & 91.4 & 80.0 & $2.67(0.98-7.25)$ & & 91.4 & 20.0 & 53.3 & $0.76(0.86 ; 0.68)$ & $0.75(0.80 ; 0.70)$ \\
\hline Squamous metaplasia & 0.0 & 17.5 & $0.000(0.000--)$ & & 100.0 & 17.5 & 56.0 & $0.92(1.00 ; 0.86)$ & $0.95(1.00 ; 0.90)$ \\
\hline Hyperplasia & 10.0 & 61.3 & $0.07(0.03-0.17)$ & 16.95 & 90.0 & 61.3 & 74.7 & $0.80(0.84 ; 0.77)$ & $0.88(0.95 ; 0.80)$ \\
\hline Goblet cells & 54.3 & 43.8 & $1.53(0.80-2.91)$ & & 54.3 & 56.3 & 55.3 & $0.74(0.73 ; 0.75)$ & $0.68(0.70 ; 0.65)$ \\
\hline BM thickening & 74.3 & 71.3 & $1.17(0.57-2.40)$ & & 74.3 & 28.8 & 50.0 & $0.71(0.78 ; 0.66)$ & $0.78(0.80 ; 0.75)$ \\
\hline Inflammatory infiltrate & 91.4 & 65.0 & $5.74(2.21-14.92)$ & 8.88 & 91.4 & 35.0 & 61.3 & $0.72(0.84 ; 0.62)$ & $0.73(0.80 ; 0.65)$ \\
\hline Eosinophils & 47.1 & 20.0 & $3.57(1.73-7.34)$ & 3.97 & 47.1 & 80.0 & 64.7 & $0.71(0.58 ; 0.83)$ & $0.80(0.60 ; 1.00)$ \\
\hline Smooth muscle & 87.1 & 77.5 & $1.97(0.82-4.72)$ & 6.34 & 87.1 & 22.5 & 52.7 & $0.77(0.83 ; 0.71)$ & $0.93(0.85 ; 1.00)$ \\
\hline Glands & 10.0 & 43.8 & $0.14(0.06-0.35)$ & 8.66 & 90.0 & 43.8 & 65.3 & $0.89(0.89 ; 0.89)$ & $0.93(1.00 ; 0.85)$ \\
\hline Sub mucosal fibrosis & 14.3 & 78.8 & $0.04(0.02-0.11)$ & 19.98 & 85.7 & 78.8 & 82.0 & $0.72(0.77 ; 0.68)$ & $0.78(0.65 ; 0.90)$ \\
\hline
\end{tabular}

* Repeatability and agreement data presented for asthma and COPD together and separately (asthma; COPD). ORs $>1$ indicate a positive association with the presence of asthma; OR $<1$ indicate a positive association with the presence of COPD. Sensitivity and specificity for asthma are calculated for the reported presence of variables with OR $>1$. Sensitivity and specificity for COPD are calculated for the reported presence of variables with OR $<1$. Significant $(p<0.05)$

Wald values were derived from logistic regression analyses on the concordant diagnosis of asthma and COPD (Table S4) 
Box 2

\section{Abnormal presence \\ of pathological criteria \\ in subjects aged $>45$ years \\ with $>10$ pack-years smoking \\ and post-bronchodilator $\mathrm{FEV}_{1} / \mathrm{FVC}<0.7$}

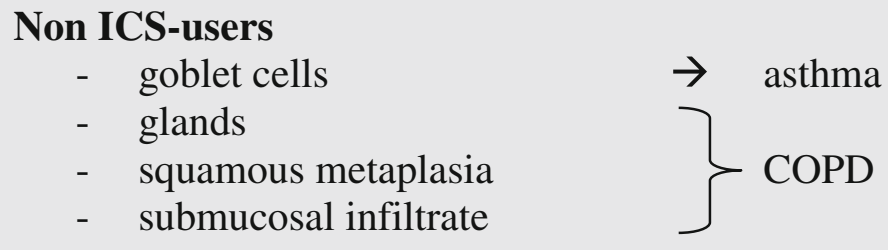

\section{ICS-users}

- basement membrane thickening

- submucosal infiltrate

- eosinophils

- glands

This study has a few limitations. First, one can argue whether or not a physician's diagnosis of asthma or COPD is an adequate gold standard. Importantly, patient charts of many years back showed that even patients with asthma and fixed airway obstruction at current investigation had reversible airway obstruction at a younger age. After accepting that our physician's diagnosis is reliable, one might still argue that the selected cases are not the ones that normally would need a pathological examination, because the clinical diagnosis was already established with certainty. Unfortunately, we have no biopsies available to compare cases with certain versus uncertain clinical diagnosis. Our aim however was to establish clues at the microscopical level with strong relation with either asthma or COPD, to be used as clues in difficult biopsies to direct towards a likely diagnosis. Secondly, one could argue that biopsies were not examined by microscope but on screen, which reflects but is not identical to the real-life situation in daily diagnostic practice. This indeed should be validated as is indicated by a recent guideline [19]. Nevertheless, in our approach, all pathologists were exposed to identical images, which improved standardization and allowed pathologists to scroll easily through complete high-resolution slides and varying magnification as in classical microscopy. Third, we did not include slides from healthy controls or from subjects with another lung disease. Consequently, the terms sensitivity and specificity only refer to asthma and COPD, compatible with our study question whether a pathologist is able to give a concordant diagnosis of asthma or COPD. We therefore consider our study design appropriate.

In conclusion, we show that the differentiation between asthma and COPD, based on histopathological examination only of a bronchial biopsy, without adequate clinical information, is difficult. This contrasts with the high percentage of concordant diagnosis observed for a number of reported pathological criteria. We postulate that the diagnostic value is likely to improve when selected pathological criteria (Box 2) are applied and adequate information is provided with respect to the use of ICS. Prospective studies incorporating medical decision techniques may validate algorithms that take these issues into account.

\section{Compliance with ethical standards}

Sources of funding This study was supported by Rijksuniversiteit Groningen (University of Groningen) and Graduate School for Drug Exploration (GUIDE). 
Conflict of interest The authors declare that they have no competing interests.

Ethical approval Study protocol involving human samples was approved by the Medical Ethics Review Committee (METc) of the University Medical Center Groningen. All procedures conformed to the ethical standards of the responsible committee on human sample experimentation and performed according to the appropriate guidelines.

Informed consent Informed consent was obtained from all patients during the original studies.

Open Access This article is distributed under the terms of the Creative Commons Attribution 4.0 International License (http:// creativecommons.org/licenses/by/4.0/), which permits unrestricted use, distribution, and reproduction in any medium, provided you give appropriate credit to the original author(s) and the source, provide a link to the Creative Commons license, and indicate if changes were made.

\section{References}

1. Global strategy for the diagnosis, management, and prevention of chronic obstructive pulmonary disease. Global initiative for chronic obstructive pulmonary disease. Available from: http://www. goldcopd.com/. 2009

2. Global strategy for asthma management and prevention, global initiative for asthma (GINA). Available from: http://www.ginasthma. org/. 2012

3. Global initiative for asthma management and prevention and the global strategy for the diagnosis, management and prevention of chronic obstructive pulmonary disease. Available from: http:// www.goldcopd.com. 2014

4. Yawn BP (2009) Differential assessment and management of asthma vs chronic obstructive pulmonary disease. Medscape J Med 11: 20

5. Perret JL, Dharmage SC, Matheson MC, Johns DP, Gurrin LC, Burgess JA, Marrone J, Markos J, Morrison S, Feather I, Thomas PS, McDonald CF, Giles GG, Hopper JL, Wood-Baker R, Abramson MJ, Walters EH (2013) The interplay between the effects of lifetime asthma, smoking, and atopy on fixed airflow obstruction in middle age. Am J Respir Crit Care Med 187:42-48
6. Zeki AA, Schivo M, Chan A, Albertson TE, Louie S (2011) The Asthma-COPD Overlap Syndrome: A Common Clinical Problem in the Elderly. J Allergy (Cairo) 2011:861926

7. Tzortzaki EG, Proklou A, Siafakas NM (2011) Asthma in the Elderly: Can We Distinguish It from COPD? J Allergy (Cairo) 2011:843543

8. Guerra S (2005) Overlap of asthma and chronic obstructive pulmonary disease. Curr Opin Pulm Med 11:7-13

9. Kitch BT, Levy BD, Fanta CH (2000) Late onset asthma: epidemiology, diagnosis and treatment. Drugs Aging 17:385-397

10. Ulrik CS (1999) Outcome of asthma: longitudinal changes in lung function. Eur Respir J 13:904-918

11. Sin BA, Akkoca O, Saryal S, Oner F, Misirligil Z (2006) Differences between asthma and COPD in the elderly. J Investig Allergol Clin Immunol 16:44-50

12. Senhorini A, Ferreira DS, Shiang C, Silva LF, Dolhnikoff M, Gelb AF, Mauad T (2013) Airway dimensions in fatal asthma and fatal COPD: overlap in older patients. COPD 10:348-356

13. Bourdin A, Serre I, Flamme H, Vic P, Neveu D, Aubas P, Godard P, Chanez P (2004) Can endobronchial biopsy analysis be recommended to discriminate between asthma and COPD in routine practice? Thorax 59:488-493

14. Broekema M, ten Hacken NH, Volbeda F, Lodewijk ME, Hylkema MN, Postma DS, Timens W (2009) Airway epithelial changes in smokers but not in ex-smokers with asthma. Am J Respir Crit Care Med 180:1170-1178

15. Lapperre TS, Snoeck-Stroband JB, Gosman MM, Stolk J, Sont JK, Jansen DF, Kerstjens HA, Postma DS, Sterk PJ (2004) Dissociation of lung function and airway inflammation in chronic obstructive pulmonary disease. Am J Respir Crit Care Med 170:499-504

16. Willemse BW, ten Hacken NH, Rutgers B, Lesman-Leegte IG, Timens W, Postma DS (2004) Smoking cessation improves both direct and indirect airway hyperresponsiveness in COPD. Eur Respir J 24:391-396

17. Broekema M, Timens W, Vonk JM, Volbeda F, Lodewijk ME, Hylkema MN, ten Hacken NH, Postma DS (2011) Persisting remodeling and less airway wall eosinophil activation in complete remission of asthma. Am J Respir Crit Care Med 183:310-316

18. Meagher LC, Cousin JM, Seckl JR, Haslett C (1996) Opposing effects of glucocorticoids on the rate of apoptosis in neutrophilic and eosinophilic granulocytes. J Immunol 156:4422-4428

19. Pantanowitz L, Sinard JH, Henricks WH, Fatheree LA, Carter AB, Contis L, Beckwith BA, Evans AJ, Lal A, Parwani AV (2013) Validating whole slide imaging for diagnostic purposes in pathology: guideline from the College of American Pathologists Pathology and Laboratory Quality Center. Arch Pathol Lab Med 137:1710 1722 